\title{
TREM-2 negatively regulates LPS-mediated inflammatory response in rat bone marrow-derived MSCs
}

\author{
PEI YE ${ }^{1,2^{*}}$, DAWEI XU ${ }^{1 *}$, JINHUANG XU ${ }^{3}$, GUIWANG LIU $^{1}$, SHENGHUI HUANG ${ }^{1}$, \\ WEIQIONG ZHANG ${ }^{3}$, PEIZHONG ZHENG ${ }^{3}$, JIANHUA $\mathrm{LI}^{4}$ and JIANRONG HUANG ${ }^{1,3}$ \\ ${ }^{1}$ Department of Orthopedics, Sun Yat-sen Memorial Hospital of Sun Yat-sen University, Guangzhou, \\ Guangdong 510120; ${ }^{2}$ Department of Orthopedics, Nanhai Affiliated Hospital of Southern Medical \\ University, Foshan 528000; ${ }^{3}$ Department of Orthopedics, Zengcheng People's Hospital of Guangzhou, \\ Guangzhou, Guangdong 511300; ${ }^{4}$ Key Laboratory of Protein Modification and Degradation, School of Basic \\ Medical Sciences, Guangzhou Medical University, Guangzhou, Guangdong 510182, P.R. China
}

Received December 28, 2016; Accepted July 27, 2017

DOI: $10.3892 / \mathrm{mmr} .2017 .7212$

\begin{abstract}
To the best of our knowledge, our previous study demonstrated the expression of triggering receptor expressed on myeloid cells 2 (TREM-2) in human bone marrow mesenchymal stem cells (MSCs) for the first time. However, the inflammation regulatory role of TREM-2 in MSCs remain elusive. The aim of the present study was to investigate the immune regulation and the underlying mechanism of TREM-2 in rat bone marrow MSCs. MSCs were divided into three

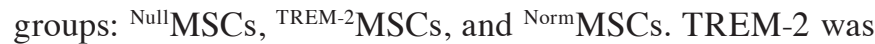
expressed in MSCs at the mRNA and protein level. Following stimulation by lipopolysaccharide (LPS), the gene transcription levels of TREM-2 and inflammatory cytokines were increased. The expression levels of inflammatory cytokines, including tumor necrosis factor- $\alpha$ (TNF- $\alpha$ ) and interleukin- $1 \beta$ (IL-1 $\beta$ ), in the ${ }^{\text {TREM-2}}$ MSCs lentiviral vector group were significantly downregulated, and the expression of IL-10 was significantly upregulated compared with the controls. Western blot analysis revealed that TREM-2 downregulated the LPS-induced inflammatory response in MSCs, which was probably associated with regulating AKT serine/threonine
\end{abstract}

Correspondence to: Professor Jianrong Huang, Department of Orthopedics, Sun Yat-sen Memorial Hospital of Sun Yat-sen University, 135 XinGangxi Road, Guangzhou, Guangdong 510120, P.R. China

E-mail: guke16@163.com

Professor Jianhua Li, Key Laboratory of Protein Modification and Degradation, School of Basic Medical Sciences, Guangzhou Medical University, Dongfeng Road West, Guangzhou, Guangdong 510182, P.R. China

E-mail:1ijianh@hotmail.com

${ }^{*}$ Contributed equally

Key words: triggering receptor expressed on myeloid cells 2, inflammation, mesenchymal stem cells kinase and p38 mitogen-activated protein kinase downstream signaling proteins. The results of the current study demonstrated that TREM-2 negatively regulates the LPS-mediated inflammatory response in MSCs suggesting that TREM-2 is a potential target of immune regulation in rat MSCs.

\section{Introduction}

Mesenchymal stem cells (MSCs) are multipotent progenitor cells that can differentiate into a variety of cell types. In addition, MSCs exert extensive immunomodulatory effects and show versatile functions in tissue engineering and immune regulation (1-3), such as inhibiting $\mathrm{T}$ cell proliferation, suppressing both proliferation and differentiation of $\mathrm{B}$ cells, and regulating the activity of natural killer cells as well as the maturity and function of dendritic cells $(4,5)$. It is widely accepted that MSCs play a role in immune regulation by secreting factors including indoleamine 2,3-dioxygenase (IDO), nitric oxide (NO), human leucocyte antigen-G (HLA-G), prostaglandin E2 (PGE2), transforming growth factor- $\beta$ (TGF- $\beta$ ), heme oxygenase-1 (HO-1), hepatocyte growth factor (HGF) and galectin-1 (6). Different cytokines probably play diverse roles during the process of immune regulation in MSCs.

Triggering receptors expressed by myeloid cells (TREM) were found to be part of an immune pattern receptor family in 2000 (7). TREM-2 is widely expressed in macrophages, dendritic cells, microglia and osteoclasts. (8-10). TREM-2 has been identified as a risk factor for Alzheimer's disease (AD) $(11,12)$. TREM-2 participates in regulating inflammation, cell differentiation and maturity, tissue repair, bone reconstruction and absorption (13-15). Peritoneal macrophages from the TREM-2 gene knockout mouse produces increased TNF- $\alpha$ and IL-6 in response to LPS (16). TREM-2 negatively regulates dendritic cell and macrophage function in the presence of TLR ligands derived from bacteria and viruses, such as LPS and CpG DNA $(17,18)$. TREM-2 is responsible for the DAP12-mediated inhibition in mouse macrophages (19). However, overexpression of TREM-2 in the microglia significantly decreases the production of TNF- $\alpha$ (20). TREM-2 has drawn considerable attention due to its role in inflammation 
regulation (21). Interestingly, a recent study by our group detected the expression of TREM-2 in human bone marrow MSCs for the first time (22).

Under specific infectious status, the pro-inflammatory capability of MSCs with expression of pro-inflammatory factors has previously described (23). In our previous studies, an upregulated secretion of inflammatory cytokine were presented by silencing TREM-2 expression in MSCs. However, the inflammation regulatory functions of TREM2-overexpressed MSCs remain to be studied. In the present study, MSCs from rat bone marrow were transfected with TREM-2 via lentiviral vector to investigate the regulatory effects and potential mechanisms in a lipopolysaccharide (LPS)-mediated inflammatory response.

\section{Materials and methods}

Ethics statement. The study was approved by the research Ethics Committee of Sun Yat-sen Memorial Hospital of Sun Yat-sen University. All animals care and experimentation were approved by the experimental animal center of Sun Yat-sen university [license no. SCXK (Yue) 2011-0029]. Efforts were made to reduce the number of animals used and minimize animal suffering. All surgery was performed under anesthesia by an intraperitoneal injection of sodium pentobarbital. All rats were sacrificed by anesthesia.

Animals. All animals were handled in compliance with the China Animal Welfare Legislation, and the protocols were approved by the Ethics Committee on the Care and Use of Laboratory Animals in Sun Yat-sen University (Guangzhou, China). Four-week-old Sprague Dawley (SD) rats were purchased from the experimental animal center of Sun Yat-sen University (Guangzhou, China). The animals were housed in the specific pathogen-free class animal room with standard humidity, temperature and illumination circle hours $(50 \pm 5 \%$; $\left.23 \pm 1^{\circ} \mathrm{C} ; 12 \mathrm{~h}\right)$. All animals had access to water and food freely throughout the experiments.

Reagents and antibodies. Fetal bovine serum, low-glucose Dulbecco's Modified Eagle's medium (L-DMEM), osteoblast, adipocyte and chondrocyte differentiation culture solutions were purchased from Gibco (Grand Island, NY, USA). Alizarin red, Alcian blue and oil red O staining kits were purchased from Sigma-Aldrich (St. Louis, MO, USA). CD44, CD90, CD29, CD34, CD14 and CD45 primary antibodies were provided by BD Biosciences (San Jose, CA, USA). TREM-2 primary antibody was purchased from Santa Cruz Biotechnology, Inc. (Santa Cruz, CA, USA). Goat anti-rabbit immunofluorescence kit (secondary antibody) was supplied by ZSGB-Bio Company (Beijing, China). Reverse transcription polymerase chain reaction (RT-PCR) kits were purchased from Takara Bio (Dalian, China). LPS, TREM-2 lentiviral solution, and Enzyme-linked immunosorbent assay (ELISA) kits for rat TNF- $\alpha$, IL-1 $\beta$ and IL-10 were purchased from Sigma-Aldrich. Primer synthesis was performed by Invitrogen Life Technologies, Shanghai, China. Puromycin was purchased from Abcam (Cambridge, MA, USA); AKT, p-AKT, p38 MAPK, p-p38 MAPK, ERK1/2, p-ERK1/2 and $\mathrm{p}-\mathrm{NF}-\kappa \mathrm{B}$ p65 were purchased from Cell Signaling Technology, Inc. (Beverly, MA, USA).
Isolation and culture of rat MSCs. MSCs from SD rats were isolated and cultured as previously described. In brief, bilateral femur and tibia were removed under sterile conditions. After irrigated repeatedly by L-DMEM culture medium, the douche was collected. Cells were digested in $0.25 \%$ Trypsin-EDTA for continuous passage until $90 \%$ confluence was obtained. The differentiation of these cells into osteoblasts, adipocytes and chondrocytes was validated. Surface antigen of MSCs was assessed by flow cytometry and prepared for subsequent experiments (24).

RT-PCR. The obtained cells samples were collected and supplemented with $1 \mathrm{ml}$ of TRIzol reagent according to the manufacturers' instructions. The synthesis of PCR primer was performed by Invitrogen Life Technologies, as illustrated in Table I. The ratio of product and internal standard gray scale was calculated using Image J software.

Immunofluorescence and confocal microscopy. After the culture medium was aspirated, the cells were irrigated by phosphate buffer saline (PBS) 3 times, fixed in ice methanol for $10 \mathrm{~min}$, soaked in $0.3 \%$ Triton $\mathrm{X}-100$ for $10 \mathrm{~min}$ at room temperature, blocked in goat serum for $30 \mathrm{~min}$ at room temperature, blocked in primary antibody at $4{ }^{\circ} \mathrm{C}$ overnight and rewarmed for $30 \mathrm{~min}$ the next day. Next, the cells were incubated with secondary antibody labeled with green fluorescence and nuclear stained by 4'6-diamidino-2-phenylindole (DAPI) and mounted in 5\% glycerin for microscopic examination under immunofluorescence and confocal microscope. The fluorescent expression of TREM-2 and subcellular localization were observed.

Western blot analysis. The protein concentration was measured by bicinchoninic acid assay and subjected to SDS-PAGE gel electrophoresis and transferred onto the polyvinylidene fluoride (PCDF) membrane, which was then blocked in $5 \%$ skimmed milk powder for $1 \mathrm{~h}$. Primary antibody was added at $4^{\circ} \mathrm{C}$ overnight and washed in Tris-buffered saline and Tween 20 (TBST); incubated with secondary antibody for $1 \mathrm{~h}$ at room temperature and protein detection by ECL kit. The ratio of product and internal standard gray scale was calculated using ImageJ software.

Validating MSCs transfected with over-expressed lentiviral plasmid. The third-passage MSCs were inoculated cells and divided into three groups: Blank plasmid-transfected

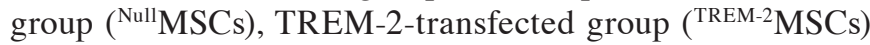
and controls ( $\left.{ }^{\mathrm{Norm}} \mathrm{MSCs}\right)$. The MSCs were transfected with blank or lentiviral plasmids carrying the TREM-2 gene, supplemented with LV-5-GFP-TREM-2 (MOI=200) and polybrene at a concentration of $5 \mu \mathrm{g} / \mathrm{ml}$, cultured at $37^{\circ} \mathrm{C}$ and $5 \% \mathrm{CO}_{2}$, and cultured for another $24 \mathrm{~h}$ with fresh culture solution. At $72 \mathrm{~h}$, the cells were observed under fluorescence microscope and the expression of GFP and the transfection efficiency was measured. The cell lines stably expressing target gene were screened by puromycin. The morphology and proliferation of MSCs after transfection were detected by 3-(4,5-Dimethylthiazol-2-yl)-2,5-diphenyltetrazolium bromide (MTT) assay according to the MTT assay manufacturer's instructions in 1, 3, 5, 7 and 9 days. 
Table I. Primers used for RT-PCR.

Gene (accession no.)

Primer sequence

Product size (bp)

TREM-2 (NM_001106884.1)

TNF- $\alpha$ (NM_012675.3)

IL-1 $\beta$ (NM_031512.2)

IL-10 (NM_012854.2)

$\beta$-actin (NM_031144.3)
5'-TCCTGTTGCTGGTCACAGAG-3'

5'-CTCCCATTCTGCTTCCTCAG-3'

5'-CCACGTCGTAGCAAACCACCAAG-3'

5'-CAGGTACATGGGCTCATACC-3'

5'-CTGTGACTCGTGGGATGATG-3'

5'-GGGATTTTGTCGTTGCTTGT-3'

5'-CCTGCTCTTACTGGCTGGAG-3'

5'-TGTCCAGCTGGTCCTTCTTT-3'

5'-GGAGATTACTGCCCTGGCTCCTA-3'

5'-GACTCATCGTACTCCTGCTTGCTG-3'
220

319

210

350

150

RT-PCR, reverse transcription polymerase chain reaction; TREM-2, triggering receptor expressed on myeloid cells 2; TNF, tumor necrosis factor; IL, interleukin.

ELISA. After $1 \mu \mathrm{g} / \mathrm{ml}$ LPS stimulation for $6 \mathrm{~h}$, the supernatant of the culture solution was collected and the levels of TNF- $\alpha$, IL-1 $\beta$ and IL-10 proteins of the solution were assessed by ELISA according to the manufacturer's instructions.

Statistical analysis. All data were analyzed by SPSS 20.0 statistical software (SPSS Inc., Chicago, IL, USA). Measurement data are expressed as the mean \pm standard deviation (SD). Statistical comparison between two groups was analyzed by t-test. Statistical comparison among multiple groups was performed using one-way ANOVA. $\mathrm{P}<0.05$ was considered to indicate a statistically significant difference.

\section{Results}

Isolation and culture of rat bone marrow-derived MSCs. Rat bone marrow MSCs were isolated from the whole bone marrow. When the medium was replaced for the first time at $24 \mathrm{~h}$, a slight amount of MSCs were allowed to attach. Along with the medium replacement and cell passage, the cells were gradually purified and grew in a shuttle and spiral pattern (Fig. 1Aa). Following osteogenic differentiation of MSCs for 21 days, Alizarin red staining revealed the formation of calcified nodes (Fig. 1Ab). Alcian blue staining at 14 days for chondrogenic differentiation indicated the MSCs were differentiated into cartilage-like cells (Fig. 1Ac). Oil red $\mathrm{O}$ staining revealed the formation of lipid droplet and vacuolization at 7 days of adipogenic induction (Fig. 1Ad). Flow cytometry was performed to detect the surface antigens of the third-passage MSCs and indicated the following: CD44 (99.35\%), CD90 (99.06\%), CD29 (99.83\%), CD34 (0.58\%), CD14 (1.34\%) and CD45 (0.72\%). These results demonstrated that the isolation and culture of MSCs were successful and qualified for subsequent experiments.

The expression of TREM-2 in MSCs. RT-PCR analysis revealed that TREM-2 mRNA was expressed in MSCs (Fig. 1C). Furthermore, western blot analysis confirmed the expression of TREM-2 protein in MSCs (Fig. 1D). Confocal microscopy was performed to further locate the expression of TREM-2 protein in MSCs (Fig. 1E).

Effects of LPS on TNF- $\alpha, I L-1 \beta$ and TREM-2 mRNA. TREM-2 mRNA expression in rat MSCs changed with the duration of LPS stimulation (Fig. 2A). TREM-2 expression peaked at $6 \mathrm{~h}$ after LPS treatment $(\mathrm{P}<0.05)$ (Fig. 2B). The expression levels of TNF- $\alpha$ and IL-1 $\beta$ mRNA at 2,4 and $6 \mathrm{~h}$ were significantly higher than those in controls (both $\mathrm{P}<0.05$ ) (Fig. 2C and D).

Transfection of TREM-2 recombinant lentiviral plasmid in MSCs. After $72 \mathrm{~h}$ of transfection, fluorescence microscopy was used to observe the expression and cell status of MSCs (Fig. 3A). Fluorescent expression of MSCs was reduced with <200 MOI. Expression fluorescence intensity of MSCs increased significantly at an MOI of 200 and the solution did not lead to cell death or affect normal cell growth. However, cell death occurred over $200 \mathrm{MOI}$. Thus, the optimal MOI of MSCs transfected with TREM-2 was 200.

MTT assay revealed no significant differences in cell

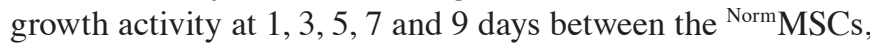

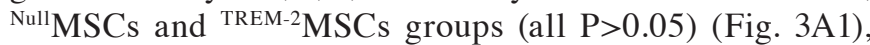
suggesting that TREM-2 and blank lentiviral vectors exerted no effect upon the growth activity of MSCs.

RT-PCR showed that the expression level of TREM-2 mRNA was $1.99 \pm 0.90$ in the ${ }^{\text {TREM-2}}$ MSCs group, which was significantly higher than $0.32 \pm 0.04$ and $0.38 \pm 0.05$ in the

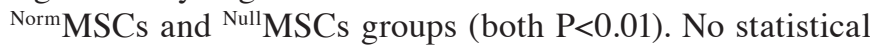

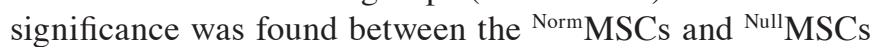
groups ( $\mathrm{P}>0.05)$, as illustrated in Fig. 3A3-4.

The expression of TREM-2 protein in MSCs was detected by western blot analysis (Fig. 3A5-6); a protein band at $26 \mathrm{kD}$ was observed in each group. The relative value of protein was $0.61 \pm 0.26$ in the ${ }^{\text {TREM-2}}$ MSCs group, which was significantly higher than $0.28 \pm 0.14$ in the NullMSCs group and $0.23 \pm 0.08$

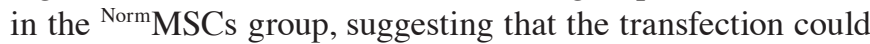
significantly increase the expression level of TREM-2 protein in MSCs. 
A

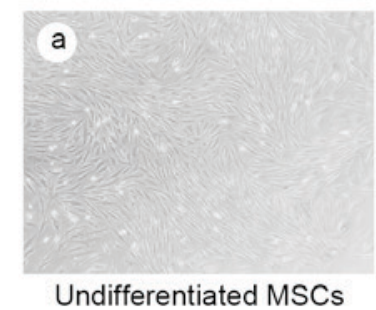

B
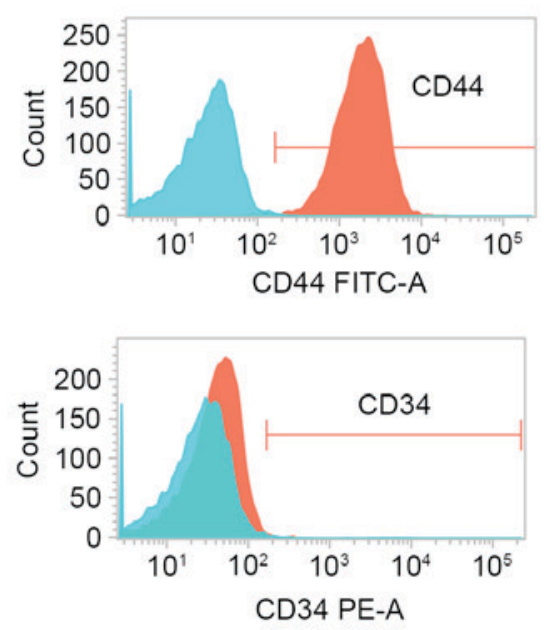

C
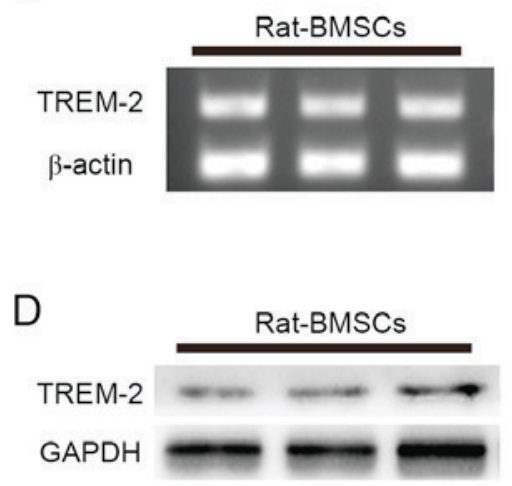

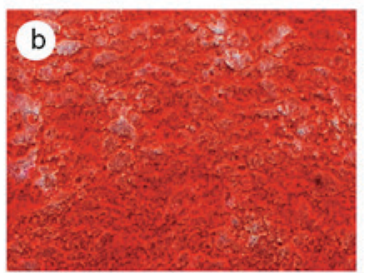

Osteogenic differentiation

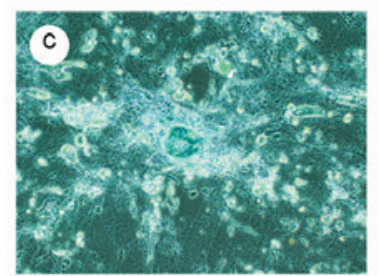

Chondrogenic differentiation

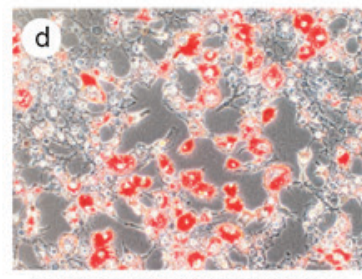

Adipogenic differentiation
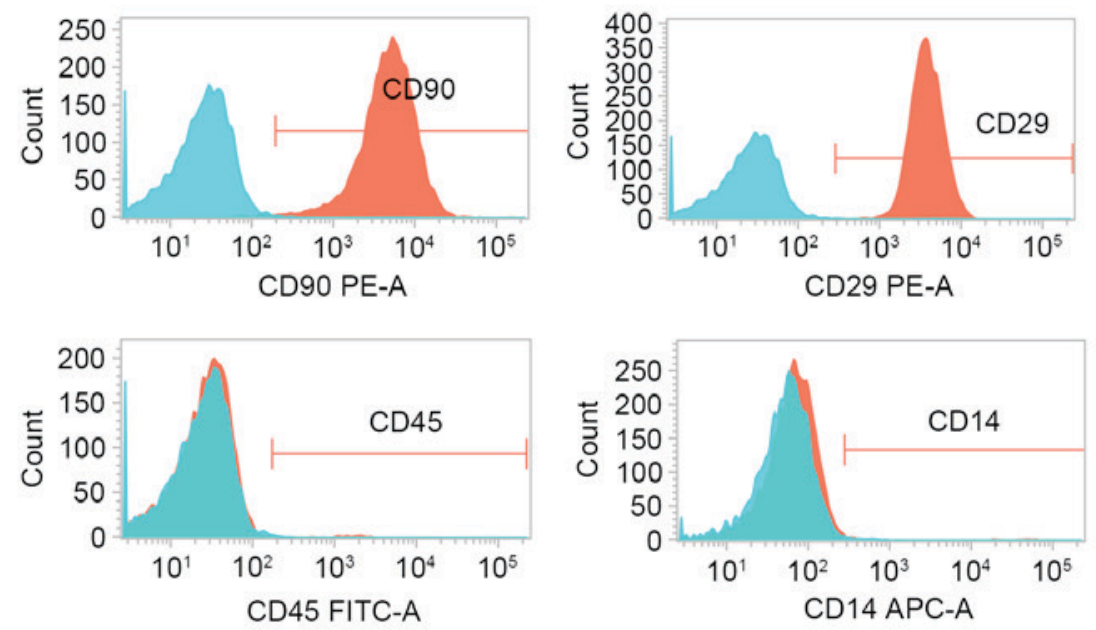

E
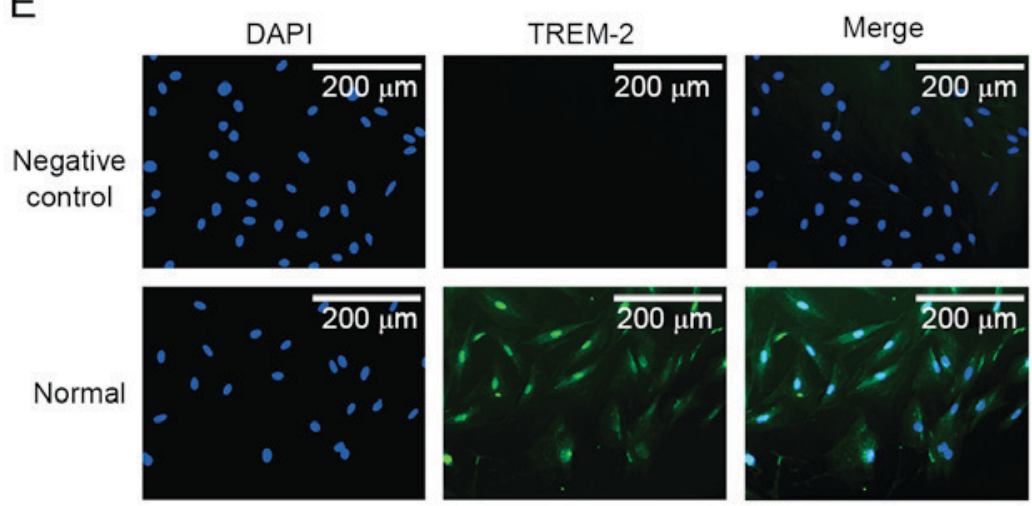

Figure 1. Transfection efficiency and growth activity of MSCs transfected with TREM-2. (Aa) The third passage undifferentiated MSCs were inoculated at a density of $5 \times 10^{6}$ cells $/ \mathrm{ml}$ and MSCs grew in a unicellular cluster cloning pattern. (Ab) The osteogenic ability and a substantial amount of sulfating proteoglycan was aggregated. (Ad) The adipogenic ability at 7 day culture was assessed and changes were observed. Original magnification: x16 for Aa; x100 for Ab-d. (B) Flow cytometry was used to detect the surface antigens of MSCs: CD44 (99.35\%), CD90 (99.06\%), CD29 (99.83\%), CD34 (0.58\%), CD14 (1.34\%) and CD45 (0.72\%). (C) The expression of TREM-2 mRNA in MSCs was detected by RT-PCR (n=3). (D) The expression of TREM-2 protein in MSCs was detected by western blot analysis ( $\mathrm{n}=3$ ). (E) The expression of TREM-2 protein in MSCs was detected by immunofluorescence staining with anti-TREM-2 antibody. The nuclei were counterstained with DAPI. Original magnification: x400 for all micrographs. MSCs, mesenchymal stem cells; TREM-2, triggering receptor expressed on myeloid cells 2; RT-PCR, reverse transcription polymerase chain reaction.

TREM-2 downregulates LPS-mediated expression of inflammatory cytokines and enhances anti-inflammatory factor expression. ELISA revealed that the expression levels of TNF- $\alpha$ and IL-1 $\beta$ protein were significantly elevated $6 \mathrm{~h}$ after LPS stimulation (both $\mathrm{P}<0.05$ ) (Fig. $\mathrm{C} 1$ and $\mathrm{C} 2$ ), whereas the level of IL-10 protein was not considerably altered (Fig. 3C3). The results of RT PCR finding were consisted with the protein levels (data not shown).

Altered expression of downstream signaling molecules AKT and p38MAPK after TREM-2 transfection. Effects of LPS stimulation at different time points on the expression of phosphory protein in MSCs were not shown. Phosphorylated and total protein levels of AKT, p38 MAPK, ERK1/2 and NF- $\kappa$ B in the ${ }^{\text {Null }}$ MSCs and ${ }^{\text {TREM-2}}$ MSCs were examined by western blot analysis before and after LPS stimulation for $15 \mathrm{~min}(1 \mu \mathrm{g} / \mathrm{ml})$ (Fig. 4A1 and B1). Western blot analysis revealed significantly elevated phosphorylation of AKT in the LPS-stimulated

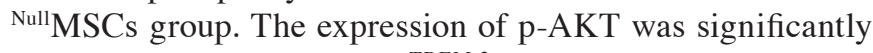
higher in LPS stimulated ${ }^{\text {TREM-2}}$ MSCs group than that in

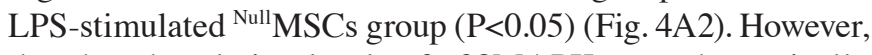
the phosphorylation levels of p38MAPK were dramatically 
A

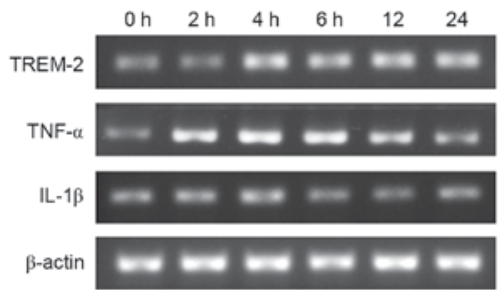

C

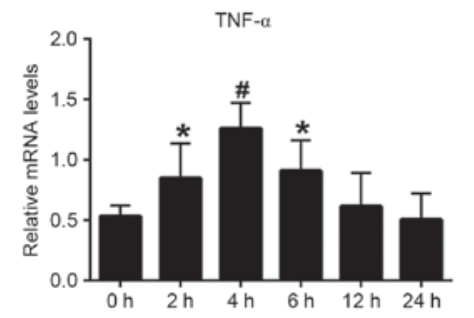

B

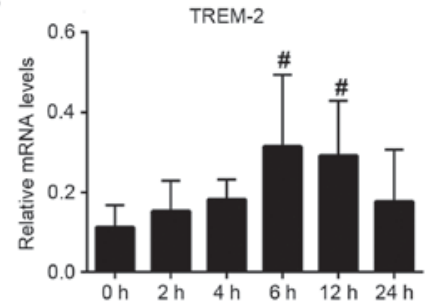

D

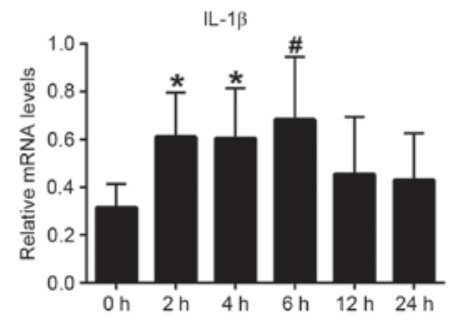

Figure 2. Expression of TREM-2 and cytokines in LPS-stimulated MSCs at different time points. (A) The changes in the expression of TREM-2, TNF- $\alpha$ and IL-1 $\beta$ mRNA in MSCs under LPS stimulation at $0,2,4,6,12$ and $24 \mathrm{~h}$ were assessed by RT-PCR. The transcriptional levels of (C) TNF- $\alpha$ and (D) IL-1 $\beta$ at 2, 4 and $6 \mathrm{~h}$ were significantly enhanced, whereas the transcriptional level of (B) TREM-2 peaked at $6 \mathrm{~h}$ and diminished gradually afterward; "P<0.05, compared with $0 \mathrm{~h}$ control group; ${ }^{\prime} \mathrm{P}<0.01$, compared with $0 \mathrm{~h}$ control group; $\beta$-actin was amplified as a positive control ( $\mathrm{n}=5$, mean $\pm \mathrm{SD}$ ). TREM-2, triggering receptor expressed on myeloid cells 2; LPS, lipopolysaccharide; MSC, mesenchymal stem cells; TNF, tumor necrosis factor; IL, interleukin; RT-PCR, reverse transcription polymerase chain reaction.

A1
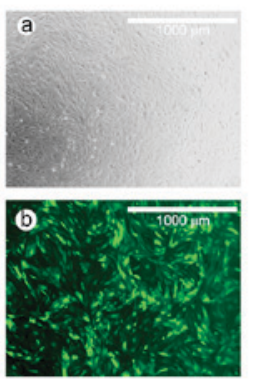

B1

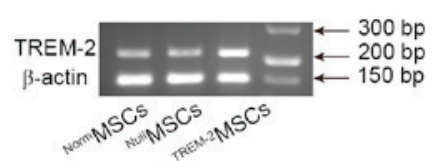

B2

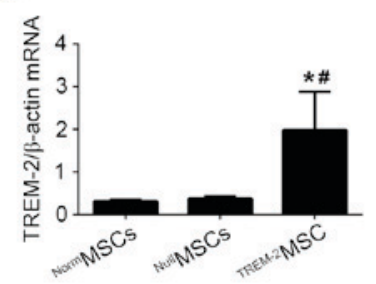

A2

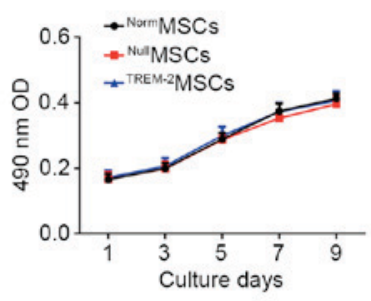

B3

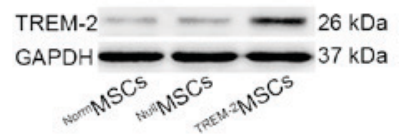

B4

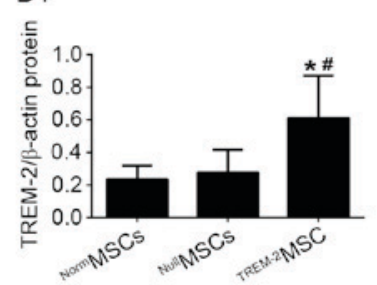

C1

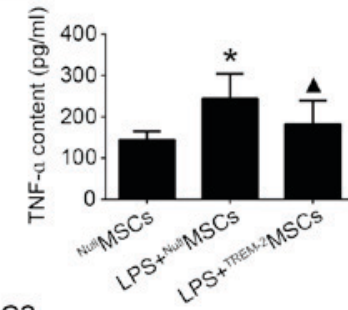

$\mathrm{C} 2$

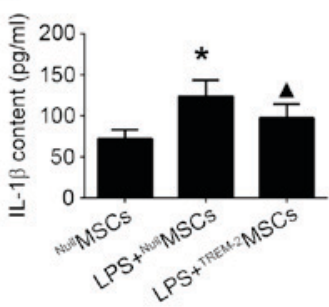

C3

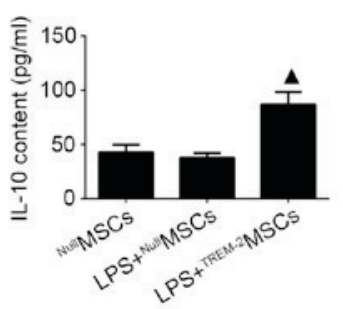

Figure 3. TREM-2 transfection efficiency and effect of TREM-2 on the expression of TNF- $\alpha$, IL-1 $\beta$ and IL-10 in LPS-treated MSCs. (A1) With MOI 200, the expression of fluorescence protein was observed at $72 \mathrm{~h}$ MSCs transfected with TREM-2 via lentiviral vector, original magnification: $\mathrm{x} 40$. (A2) The MSCs transfected with TREM-2 or blank vectors exerted no significant influence on cell growth activity by MTT assay. (B1) The expression of TREM-2 mRNA using RT-PCR with quantification (B2) in the TREM-2MSCs group was considerably elevated. (B3) The expression level of TREM-2 protein measured using western blotting and (B4) quantified, was significantly enhanced in the TREM-2MSCs group. "P<0.01, compared with the NormMSCs group; ${ }^{~} \mathrm{P}<0.01$, compared with NullMSCs group $(\mathrm{n}=7$, mean $\pm \mathrm{SD})$. The secretion of $(\mathrm{C} 1)$ TNF- $\alpha,(\mathrm{C} 2)$ IL-1 $\beta$ and $(\mathrm{C} 3)$ IL-10 proteins in LPS-stimulated MSCs was assessed by ELISA. Overexpression of TREM-2 significantly reduced the proteins of TNF- $\alpha$ and IL-1 $\beta$ but remarkably upregulated the secretion of IL-10. "P<0.01, compared with the NullMSCs group; ${ }^{\wedge} \mathrm{P}<0.05$, compared with the LPS-stimulated the NullMSCs group $(\mathrm{n}=6$, mean $\pm \mathrm{SD})$. TREM-2, triggering receptor expressed on myeloid cells 2; TNF, tumor necrosis factor; IL, interleukin; LPS, lipopolysaccharide; MSC, mesenchymal stem cells; MTT, 3-(4,5-Dimethylthiazol-2-yl)-2,5-diphenyltetrazolium bromide RT-PCR, reverse transcription polymerase chain reaction. 


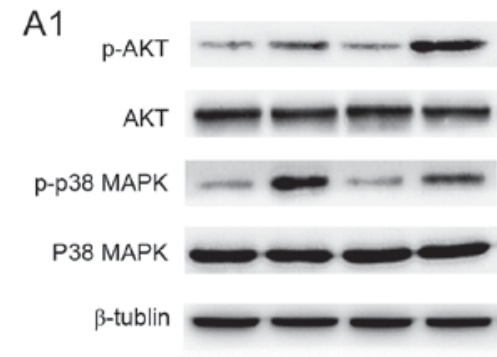

B1

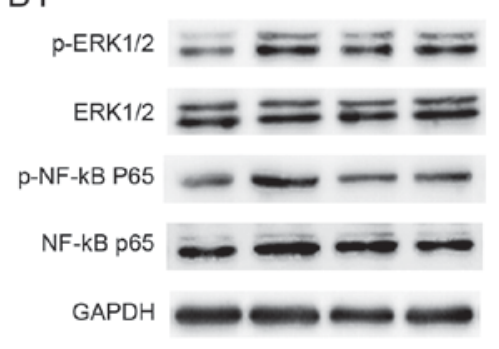

A2

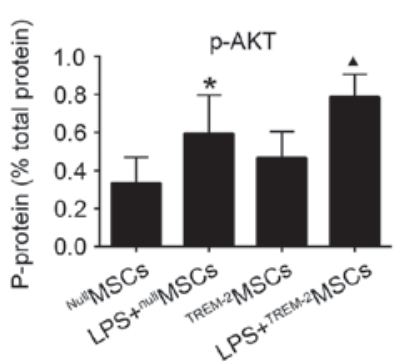

B2

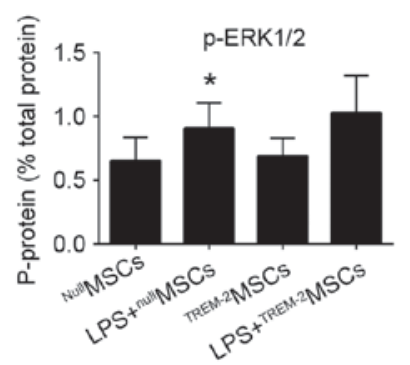

A3

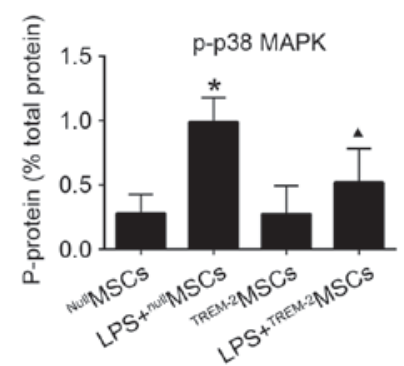

B3

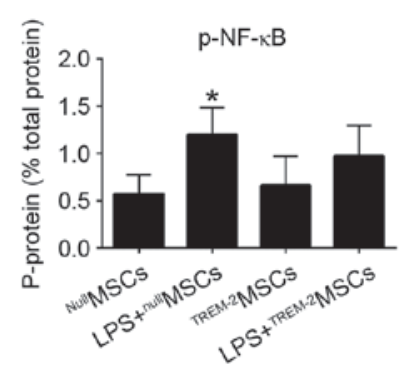

Figure 4. Protein expression of AKT, p38 MAPK, ERK1/2 and NF- $\kappa$ B in TREM-2-transfected MSCs. (A1, B1) Phosphorylated and total protein levels of AKT, p38 MAPK, ERK1/2 and NF-кB in NullMSCs and TREM-2MSCs were examined by Western blot before and after LPS stimulation for 15 min. (A2-3) Overexpression of TREM-2 enhanced downstream protein AKT and decreased p38 MAPK. (B2-3) No significant changes in phosphorylation levels of ERK1/2 and NF- $\kappa$ B were observed. ${ }^{*} \mathrm{P}<0.05$, compared with the NullMSCs group; ${ }^{\wedge} \mathrm{P}<0.05$, compared with the LPS-stimulated NullMSCs group ( $\mathrm{n}=5$, mean \pm SD).

decreased $(\mathrm{P}<0.05)$ compared to those in the LPS-stimulated

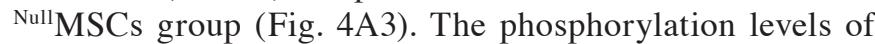
ERK1/2 and NF- $\kappa$ B p65 did not significantly differ between two groups (both $\mathrm{P}>0.05$ ) (Fig. 4B2 and B3).

\section{Discussion}

As a recently found immune pattern receptor, TREM-2 is widely expressed in macrophages, dendritic cells, microglia and osteoclasts (25). TREM-2 is a pivotal regulating factor and its expression levels in alternative types of cells continue to garner attention from scholars. In the present investigation, we confirmed that TREM-2 protein and mRNA were expressed in MSCs.

The present study showed that TREM-2 was highly expressed in MSCs. Consequently, the exact role of TREM-2 in MSCs could be elucidated by deep analysis of the influence of TREM-2 on LPS-mediated inflammatory factors. In previous studies (26), silencing of TREM-2 significantly exacerbates pro-inflammatory responses induced by LPS in HEK293 cells, and overexpression of TREM-2 reduces LPS-induced inflammatory responses. TREM-2 recombinant plasmid was constructed and successfully transfected into the MSCs. Subsequent experiments found that the transcriptional and translational levels of inflammatory cytokines TNF- $\alpha$ and IL-1 $\beta$ were elevated in LPS-stimulated MSCs, whereas the expression of TNF- $\alpha$ and IL-1 $\beta$ mRNA and the secretion of protein were reduced after TREM- 2 transfection. These results indicating that TREM-2 can block inflammatory chain reaction mediated by LPS during early stage, thereby suppressing the inflammation cascade effect. Our data demonstrated that TREM-2 exerts negative regulatory effects on inflammatory response. Moreover, TREM-2 was able to upregulate the expression levels of anti-inflammatory factor IL-10 mRNA and protein in LPS-stimulated MSCs. Considering the multiple functions of IL-10 in immune responses, it is assumed that TREM-2 could inhibit the release of inflammatory factors and decrease the antigen presentation of MSCs towards pathogenic bacteria, and possess self-limiting effect on inflammation.

In MSCs, LPS induces inflammatory response mainly through downstream signaling molecules including MAPKs, ERK1/2, JNK and P38 MAPK. Recent studies have revealed that the underlying mechanism of negative regulation of TREM-2 on the secretion of inflammatory factors is probably that TREM-2 binds to its ligand and then transduces activation signal through immune receptor tyrosine-based activation motif, which accelerates the phosphorylation of growth factor receptor-bound protein-2, activates the signaling transduction pathways of PI3K and PLC $\gamma$, provokes the changes of intracellular $\mathrm{Ca}^{2+}$ and eventually suppresses the release of pro-inflammatory factors $(16,27,28)$.

The activation of TREM-2/DAP12 complex in microglia transfected with TREM-2 arouses the phosphorylation of signaling molecule ERK1/2 (29). Moreover, the phosphorylation of ERK1/2 is associated with suppressing the inflammatory response and the phagocytosis of apoptotic cells. However, previous studies have found that the phagocytosis of external granules and bacteria is intimately correlated with the activation of downstream signaling pathway PI3K/AKT of TREM-2/DAP12 complex rather than ERK $(30,31)$. TREM-2 activates the PI3K/AKT signaling pathway, whereas ERK, JNK and P38 are not associated with the anti-inflammatory effect in keratitis patients infected with pseudomonas aeruginosa $(32,33)$. In the present study, the phosphorylated levels of downstream signaling molecules AKT, p38MAPK, ERK1/2

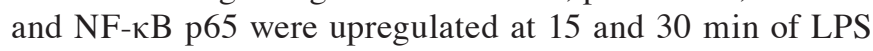


stimulation. The phosphorylated level of LPS-mediated AKT was significantly elevated in MSCs with overexpressing TREM-2, but that of p38MAPK was dramatically downregulated. The results in our study were consistent with previous findings $(30,33)$.

Clinical treatment with MSCs application may not be efficient (34). Owning to its potential dangerous pro-inflammatory capability, further research about the inflammation regulatory role of MSCs should be tabled. We demonstrated that TREM-2 was expressed in rat bone marrow MSCs. Under LPS stimulation, the expression of TREM-2 was upregulated in MSCs. Overexpression of TREM-2 significantly inhibited the production of TNF- $\alpha$ and IL-1 $\beta$ in MSCs and promoted the secretion of anti-inflammatory factor IL-10, which is probably correlated with upregulating the signaling protein AKT and downregulating the expression of p38MAPK. Our results further indicate that TREM-2 might play a vital role in the immune regulation and might serve as a potential target of immune regulation.

\section{Acknowledgements}

The present study was supported by the Science and Technology Plan Project of Guangdong Province (no. 2010B031600184), the Key Research Foundation of Guangzhou Branch letter Bureau (no. 11C31120184) and the Natural Science Foundation of Guangdong (nos. S2012010010629 and S2013010013768).

\section{References}

1. Nauta AJ and Fibbe WE: Immunomodulatory properties of mesenchymal stromal cells. Blood 110: 3499-3506, 2007.

2. Murphy MB, Moncivais K and Caplan AI: Mesenchymal stem cells: Environmentally responsive therapeutics for regenerative medicine. Exp Mol Med 45: e54, 2013.

3. Prockop DJ, Kota DJ, Bazhanov N and Reger RL: Evolving paradigms for repair of tissues by adult stem/progenitor cells (MSCs). J Cell Mol Med 14: 2190-2199, 2010.

4. Tyndall A: Mesenchymal stem cell treatments in rheumatology: A glass half full? Nat Rev Rheumatol 10: 117-124, 2014.

5. Ankrum JA, Ong JF and Karp JM: Mesenchymal stem cells: Immune evasive, not immune privileged. Nat Biotechnol 32: 252-260, 2014.

6. Dazzi F, Lopes L and Weng L: Mesenchymal stromal cells: A key player in 'innate tolerance'? Immunology 137: 206-213, 2012.

7. Bouchon A, Dietrich J and Colonna M: Cutting edge: Inflammatory responses can be triggered by TREM-1, a novel receptor expressed on neutrophils and monocytes. J Immunol 164: 4991-4995, 2000.

8. Colonna M: TREMs in the immune system and beyond. Nat Rev Immunol 3: 445-453, 2003.

9. Molloy EJ: Triggering receptor expressed on myeloid cells (TREM) family and the application of its antagonists. Recent Pat Antiinfect Drug Discov 4: 51-56, 2009.

10. Ford JW and McVicar DW: TREM and TREM-like receptors in inflammation and disease. Curr Opin Immunol 21: 38-46, 2009.

11. Jonsson T, Stefansson H, Steinberg S, Jonsdottir I, Jonsson PV, Snaedal J, Bjornsson S, Huttenlocher J, Levey AI, Lah JJ, et al: Variant of TREM2 associated with the risk of Alzheimer's disease. N Engl J Med 368: 107-116, 2013.

12. Guerreiro R, Wojtas A, Bras J, Carrasquillo M, Rogaeva E, Majounie E, Cruchaga C, Sassi C, Kauwe JS, Younkin S, et al: TREM2 variants in Alzheimer's disease. N Engl J Med 368: 117-127, 2013.

13. Paradowska-Gorycka A and Jurkowska M: Structure, expression pattern and biological activity of molecular complex TREM-2/DAP12. Hum Immunol 74: 730-737, 2013.

14. Thrash JC, Torbett BE and Carson MJ: Developmental regulation of TREM2 and DAP12 expression in the murine CNS: Implications for Nasu-Hakola disease. Neurochem Res 34: 38-45, 2009.
15. Sieber MW, Jaenisch N, Brehm M, Guenther M, Linnartz-Gerlach B, Neumann H, Witte OW and Frahm C: Attenuated inflammatory response in triggering receptor expressed on myeloid cells 2, (TREM2) knock-out mice following stroke. PLoS One 8: e52982, 2013.

16. Turnbull IR, Gilfillan S, Cella M, Aoshi T, Miller M, Piccio L, Hernandez M and Colonna M: Cutting edge: TREM-2 attenuates macrophage activation. J Immunol 177: 3520-3524, 2006.

17. Ito $\mathrm{H}$ and Hamerman JA: TREM-2, triggering receptor expressed on myeloid cell-2, negatively regulates TLR responses in dendritic cells. Eur J Immunol 42: 176-185, 2012.

18. Chang JH, Chang EJ, Kim HH and Kim SK: Enhanced inhibitory effects of a novel $\mathrm{CpG}$ motif on osteoclast differentiation via TREM-2 downregulation. Biochem Biophys Res Commun 389: 28-33, 2009.

19. Hamerman JA, Jarjoura JR, Humphrey MB, Nakamura MC, Seaman WE and Lanier LL: Cutting edge: Inhibition of TLR and FcR responses in macrophages by triggering receptor expressed on myeloid cells (TREM)-2 and DAP12. J Immunol 177: 2051-2055, 2006.

20. Takahashi K, Rochford CD and Neumann H: Clearance of apoptotic neurons without inflammation by microglial triggering receptor expressed on myeloid cells-2. J Exp Med 201: 647-657, 2005.

21. Piccio L, Buonsanti C, Mariani M, Cella M, Gilfillan S, Cross AH, Colonna $\mathrm{M}$ and Panina-Bordignon P: Blockade of TREM-2 exacerbates experimental autoimmune encephalomyelitis. Eur J Immunol 37: 1290-1301, 2007.

22. Zhang WQ, Huang SH, Huang X, Li JH, Ye P, Xu J, Zheng PZ, Shen HY and Huang JR: Regulation of human mesenchymal stem cell differentiation by TREM-2. Hum Immunol 77: 476-482, 2016.

23. Yan H, Wu M, Yuan Y, Wang ZZ, Jiang H and Chen T: Priming of Toll-like receptor 4 pathway in mesenchymal stem cells increases expression of $\mathrm{B}$ cell activating factor. Biochem Biophys Res Commun 448: 212-217, 2014.

24. Dominici M, Le Blanc K, Mueller I, Slaper-Cortenbach I, Marini F, Krause D, Deans R, Keating A, Prockop Dj and Horwitz E: Minimal criteria for defining multipotent mesenchymal stromal cells. The international society for cellular therapy position statement. Cytotherapy 8: 315-317, 2006.

25. Sharif $\mathrm{O}$ and Knapp S: From expression to signaling: Roles of TREM-1 and TREM-2 in innate immunity and bacterial infection. Immunobiology 213: 701-713, 2008.

26. Zhong L, Chen XF, Zhang ZL, Wang Z, Shi XZ, Xu K, Zhang YW, Xu H and Bu G: DAP12 stabilizes the C-terminal fragment of the triggering receptor expressed on myeloid cells-2, (TREM2) and Protects against LPS-induced Pro-inflammatory response. J Biol Chem 290: 15866-15877, 2015.

27. Ramanathan B, Minton JE, Ross CR and Blecha F: Cloning of porcine triggering receptor expressed on myeloid cells-1 (TREM-1) and its induction by lipopolysaccharide, peptidoglycan, and Salmonella enterica serovar Typhimurium infection. Dev Comp Immunol 29: 1-7, 2005.

28. Helming L, Tomasello E, Kyriakides TR, Martinez FO, Takai T, Gordon S and Vivier E: Essential role of DAP12 signaling in macrophage programming into a fusion-competent state. Sci Signal 1: ra11, 2008.

29. Takahashi K, Prinz M, Stagi M, Chechneva O and Neumann H: TREM2-transduced myeloid precursors mediate nervous tissue debris clearance and facilitate recovery in an animal model of multiple sclerosis. PLoS Med 4: e124, 2007.

30. Chen Q, Zhang K, Jin Y, Zhu T, Cheng B, Shu Q and Fang X: Triggering receptor expressed on myeloid cells-2 protects against polymicrobial sepsis by enhancing bacterial clearance. Am J Respir Crit Care Med 188: 201-212, 2013.

31. N'Diaye EN, Branda CS, Branda SS, Nevarez L, Colonna M, Lowell C, Hamerman JA and Seaman WE: TREM-2 (triggering receptor expressed on myeloid cells 2) is a phagocytic receptor for bacteria. J Cell Biol 184: 215-223, 2009.

32. Sun M, Zhu M, Chen K, Nie X, Deng Q, Hazlett LD, Wu Y, Li M, Wu M and Huang X: TREM-2 promotes host resistance against Pseudomonas aeruginosa infection by suppressing corneal inflammation via a PI3K/Akt signaling pathway. Invest Ophthalmol Vis Sci 54: 3451-3462, 2013.

33. Zhu M, Li D, Wu Y, Huang X and Wu M: TREM-2 promotes macrophage-mediated eradication of Pseudomonas aeruginosa via a PI3K/Akt pathway. Scand J Immunol 79: 187-196, 2014.

34. Waterman RS, Tomchuck SL, Henkle SL and Betancourt A: A new mesenchymal stem cell (MSC) paradigm: Polarization into a pro-inflammatory MSC1 or an Immunosuppressive MSC2 phenotype. PLoS One 5: e10088, 2010. 